\title{
A Single- vs. Multi-Item Self-Rated Health Status Measure: A 21-Country Study
}

\author{
Jeroen K. Van Ginneken* and George Groenewold
}

Netherlands Interdisciplinary Demographic Institute, The Hague, The Netherlands

\begin{abstract}
Objectives: Data have been collected on self-rated health using a single question on how individuals rate their health in cross-sectional surveys carried out in a large number of countries. Doubts have been expressed about the validity of this measure and this was the main reason to undertake the current study. Study Design: Data of 21 cross-sectional surveys were analyzed derived from the World Health Survey (WHS) carried out among adults in 2002-2003. Methods: We compared the single-item self-rated health measure with a multi-item health status index. Information on both types of measures was available from WHS. The multi-item index was constructed using data on functional limitations in daily activities. Results: The relationship of age with the multi-item health status index was linear while the relationship of age with self-rated health deviated from linearity in the younger and the oldest age groups. Both measures were compared with two criterion variables: life expectancy at age 20 and self-reported chronic conditions. The multi-item index was more strongly related to life expectancy and to chronic conditions than was the single-item self-rated health measure. Conclusions: The multi-item health status index could be a stronger predictor of mortality than the single-item self-rated health measure. It is recommended to rely in health surveys as much as possible on multi-item health status measures. Single-item self-rated health measures should continue to be used in situations where there are no other alternatives available, but researchers and policy makers should be aware of their limitations.
\end{abstract}

Keywords: Self-rated health, multi-item health status indices, validity, World Health Survey.

\section{INTRODUCTION}

Self-rated health (SRH), also called self-rated health status, self-rated general health or self-reported health is a frequently used measure of self-perceived or subjective morbidity. SRH is used in research on various topics, but in particular in longitudinal studies as a predictor of subsequent mortality [1-3]; in cross-sectional surveys as the outcome variable in studies on social inequalities in health [4-8]; and in studies on compression of morbidity [9-11].

SRH is a single-item indicator consisting of one question: "How do you rate your health? Very good, Good, Moderate, Bad or Very bad?". In some surveys more than these fiveanswer categories have been used, in others less. The wording of the answer categories has also varied starting, for example, with Excellent instead of Very good $[12,13]$. SRH belongs to the group of self-perceived morbidity measures [14]. The term self-rated health is according to us not the most suitable to use since it is nearly synonymous with selfperceived morbidity; the term self-reported general health is more appropriate as is the term single-item SRH. SRH is, however, frequently used in articles published in epidemiologic and public health journals in the past ten years and for this reason we will continue to use it. Doubts have been expressed if SRH and other types of questions or instruments in the group of self-perceived morbidity are valid and reliable measures of morbidity. It has been argued that mea-

*Address correspondence to this author at the Netherlands Interdisciplinary Demographic Institute, P.O. Box 11650, 2502 AR The Hague, The Netherlands; Tel: +31 (0)70 3565200; Fax: +31 (0)70 3647187; E-mail: Ginneken@nidi.nl sures such as self-rated health are inadequate and that instead there should be more emphasis on use of observed morbidity measures [15]. In reply, others have commented that at the societal level self-rated health could be adequate for broad assessments of health status [16].

A problem with the single-item self-rated general health question is that the answers respondents give are not only a reflection of their "objective" health status, but are also influenced by norms and expectations that individuals, groups and societies have about health. An example of such norms and expectations is the extent to which individuals at older ages compare themselves with others in the same age group. They may conclude that they are better off compared to others in this reference group even though they are actually in worse health than those at younger ages. Another example consists of cross-cultural differences in the propensity to complain about health problems. It is difficult to separate the impact of these and other cultural influences from the other factors dealing with health status. In other words, the SRH measure is a multi-dimensional concept of health status, but in the process of measuring it there is little control over the actual dimensions being tapped by respondents.

We like to emphasize that information on SRH is important in its own right as long as there is the recognition that the answers on the self-rated health question are influenced by these cultural factors. However, in practice many investigators and policy makers minimize or ignore the role of psychological and cultural factors in the process of using the self-rated health measure. It is usually taken for granted that this is a correct assumption to make and it is rarely made explicit. Here, however, we want to raise some questions about the validity of the SRH measure. 
Other measures in the group of self-perceived morbidity, apart from the single-item SRH, are multi-item functional disabilities in daily activities and symptoms of conditions or diseases. The objective of our paper is to assess how good SRH is by comparing it with a multi-item index that we constructed using information from a battery of questions dealing with various aspects of functional limitations in daily activities. In our project we developed an index that is based on various scales as applied in the World Health Survey (WHS) and we will call it the WHS Health Status Index (WHSHSI). In other words, WHSHSI is another multidimensional concept of health status, but in the process of its measurement there is specification of and control over the various dimensions that together define it. WHS surveys include both the question on SRH and a set of questions on functional limitations in daily activities. This provides us with the opportunity to compare SRH with WHSHSI. Since WHSHSI is a measure using multiple items in contrast to the single-item SRH, we expect to find that WHSHSI is a more adequate and more valid indicator of the underlying selfperceived morbidity concept than is SRH. We will test if and to what extent this is the case by using data from WHS in 21 countries.

More specifically, we will examine how in 21 countries results of WHSHSI and SRH compare with two external criterion variables. The first of these, measured at the country level, refers to the level of mortality. Attention has been drawn to the fact that interactions between selfperceived morbidity, observed morbidity and mortality are complex and vary by country and period [14]. In spite of this, there should be, at the country level, a substantial correlation between self-perceived morbidity and mortality. Therefore, we expect to find that, at the country level, WHSHSI is more strongly related to mortality (as measured with life expectancy) than is SRH. The second external criterion with which WHSHSI and SRH will be compared is, at the individual level, prevalence of a number of selfreported chronic conditions on which data were collected in WHS. Similarly, we expect to find that WHSHSI has a stronger association with a number of chronic diseases than does SRH.

\section{MATERIALS AND METHODS}

\section{Study Design and Data}

The WHO World Health Survey project consists of a number of nationally representative, cross-sectional sample surveys that were carried out in 72 countries in 2002 and 2003 in the population 18 years and older [17]. The number of respondents varied from about 500 to 7,000. Two exceptions were the Indian WHS carried out in 6 states with a sample size of about 10,000 [18] and the WHS of Mexico with nearly 39,000 respondents. The respondents were randomly selected from nationally representative sampling frames using multi-stage sampling techniques. All surveys contain questions on a number of topics that are covered in a Household and an Individual Questionnaire. Extensive pretesting of the questionnaire took place in the WHO Multicountry Survey on Health and Responsiveness in 2000-2001 [19] and in a pilot study was carried out in 12 countries in early 2002 [17]. In the various phases of implementation of the surveys attention was paid to obtain high-quality data through adequate training and close supervision of the interviewers. Monitoring of the quality of fieldwork took place by means of calculation of sample population deviation indices, response rates, rates of missing data and test-retest reliability coefficients. Details of the methodology are provided by Ustin et al. [17].

We used datasets of 21 countries from the WHS database in five regions (4 in West Europe, 4 in Central and East Europe, 5 in Latin America, 4 in Sub-Saharan Africa and 4 in Asia). The main reasons for excluding other countries were: (1) small sample size (less than 950), (2) aboveaverage non-response rates, (3) very small number of respondents 65 years and older. Within the five regions countries were selected in such a way that higher and lower mortality levels were represented. In three regions the number of countries eligible for participation corresponded to the number selected taking into account the above-mentioned exclusion criteria. In two other regions random sampling was applied to select countries in the category with high mortality levels. In Latin America we added a fifth country in order to have access to information to two lowmortality countries. Analysis of these WHS data was done for males and females 20 years and older.

\section{Outcome Variables: Two Health Status Measures}

The WHS Health Status Index (WHSHSI) and the selfrated health (SRH) measure feature in our study as the outcome variables. Questions permitting construction of WHSHSI were derived from the WHO Disability Assessment Schedule. This module used ingredients from already existing scales and instruments and was extensively pretested in 2001 and 2002. A total of 17 questions were used on the ability to carry out a number of routinely performed daily activities and on other functional limitations in the past 30 days. The questions covered topics such as mobility, self care, vision, experiencing pain, bodily discomfort, anxiety and depression. There are 5 response categories for each of these items ranging from No difficulty $(=1)$ to Extremely Difficult/Impossible $(=5)$. Principal component analysis (PCA) of the 17 items was performed in each of the 21 countries to create an interval-level variable derived from the first component scores. This first component was in all countries by far the most important with Eigenvalues of 7 and more and explaining 35 to $50 \%$ of the variance. The end result of this analysis was for each country the WHSHSI index consisting of an interval-level variable ranging from 0 (= in perfect health) to 10 (= in very bad health). The distributions of scores on this variable were skewed with the scores in less good health concentrated in the range between 9 and 10. We decided, therefore, to transform them to a dichotomous variable with the cut-off point of 9. Persons were considered to be in less good health when they had scores higher than (and including) 9.

The SRH measure is based on the already mentioned "How do you rate your health?" question. Values on this variable ranged from 1 (= Very good) to 5 (= Very bad). The distribution of scores was also skewed and this variable was therefore transformed to a dichotomous variable with a cutoff point of 3. Persons were considered to be in less good health when they said that their health was Moderate, Bad or Very bad corresponding with scores varying from 3 to (and 
including) 5. We could also have used a cut-off point of being in less good health consisting of scores 4 and 5 . However, the advantage of using scores 3-5 when using SRH is, as we will see below, that the percentages obtained are much more close to the percentages found with the scores 910 obtained with the WHSHSI measure.

\section{Criterion Variables: Life Expectancy at Age 20 and Self- Reported Conditions}

Figures on life expectancy at birth in 2000-2005 were obtained from the UN population database [20]. They were converted to data on life expectancy at age 20 by means of interpolation, using the West family of the Coale-Demeny model life table system [21].

WHS included a series of questions measuring prevalence of the following self-reported conditions: arthritis, angina pectoris, asthma, depression, tuberculosis and diabetes. The prevalence of the first five of these conditions was determined with the help of algorithms asking about symptoms occurring in the past 12 months prior to the interview. These algorithms were validated in previous research by Moussavi et al. [22]. We used the first question of the algorithms to determine the prevalence of these five chronic diseases. Respondents were regarded as positive for diabetes if they reported ever being diagnosed with this disease. Questions on diabetes were asked in 18 of the 21 countries.

\section{Statistical Methods}

Principal component analysis (PCA) was used to construct the WHSHSI interval-level variable derived from the
17 questions dealing with functional limitations. Percentage differences, Pearson regression coefficients and Spearman rank correlation coefficients were used to determine the strength of the relationships of the two health status variables with age, gender, life expectancy and chronic conditions. The $\mathrm{t}$ - and the normal test were used to determine statistical significance of differences in means and proportions. The ttest was also used to test whether the Spearman rank correlations were significantly different from zero.

The analysis was carried out in the following sequence. First, we studied age-standardized percentages in less good health obtained with WHSHSI and SRH in the 21 countries. Age-adjustment was done with the direct method using the WHO world standard population. Next, we determined whether the relationship of age with WHSHSI was similar to the relationship of age with SRH. Then, we studied the association of the age-adjusted percentages in less good health with life expectancy at age 20 . Finally, we determined the correlation between a number of chronic conditions and the two health status measures.

\section{RESULTS}

\section{Age-Adjusted Percentages in Less Good Health According to WHSHSI and SRH}

Table 1 shows age-adjusted percentages in less good health according to WHSHSI and SRH in 21 countries (men and women combined). About half of all respondents considered themselves to be in less good health using the

Table 1. Two Age-Adjusted Health Measures (WHSHSI, SRH), Age 20 +, 21 WHS Countries

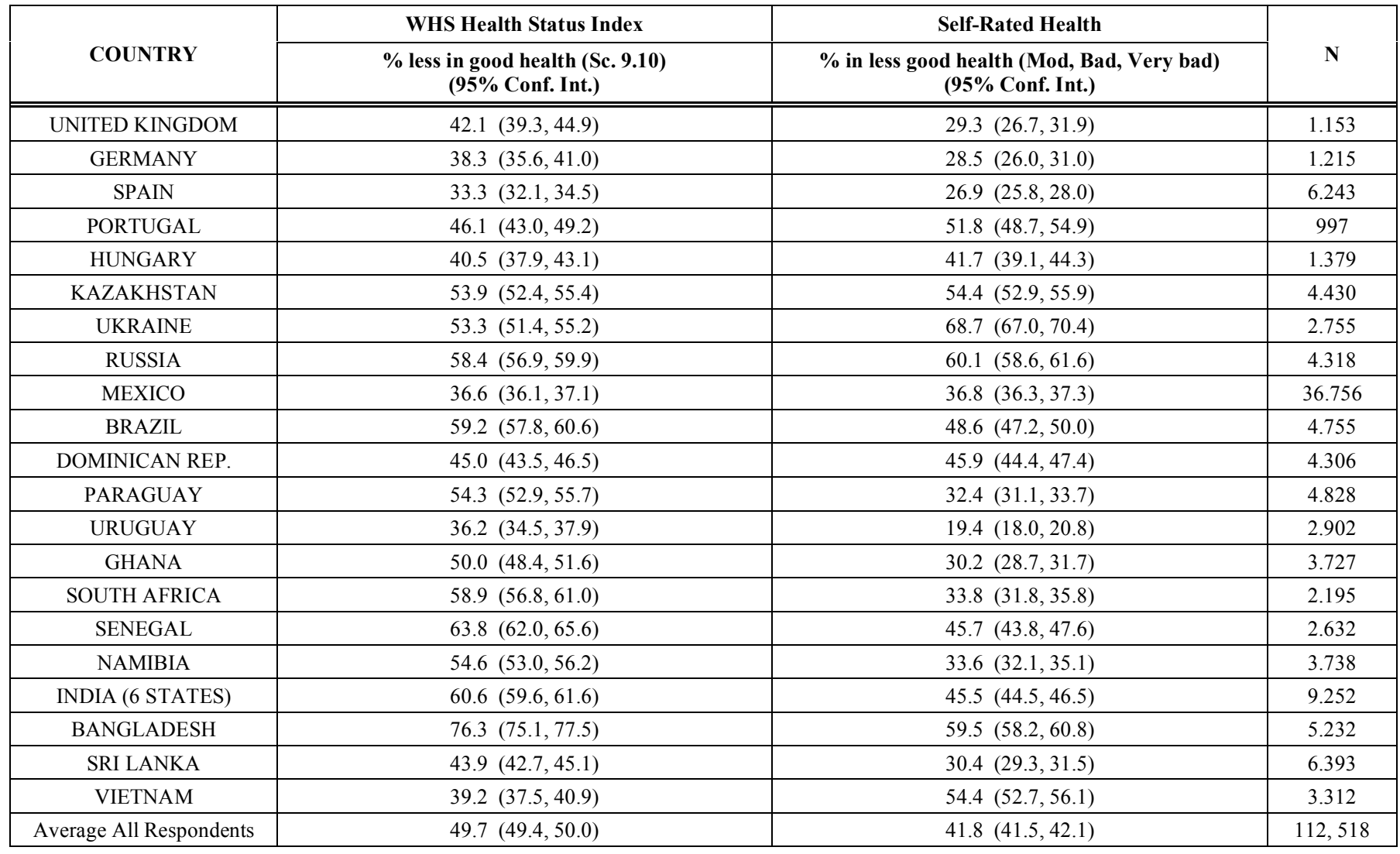


WHSHSI measure (scores 9-10). Percentages in less good health were highest in Bangladesh, India, Senegal, South Africa, Brazil and Russia and lowest in most West European countries, Hungary, Mexico, Uruguay, Sri Lanka and Vietnam. Differences in values between these countries and the overall average percentage in less good health (all 21 countries) were statistically significant. About $42 \%$ of all respondents considered themselves to be in less good health according to the SRH measure (scores 3-5). To some extent there is a different rank order of countries when SRH is used (instead of WHSHSI). According to SRH the highest rates are found in Ukraine, Russia and Bangladesh and the lowest in Uruguay and most West European countries. In all these cases, differences in scores between these countries and the overall average (all 21 countries) were statistically significant. An unusual outcome with respect to SRH was that the percentages in less good health were higher in Ukraine and Russia than in Ghana, South Africa and Namibia. We will elaborate on this anomaly in the Discussion.

Figures are also available on percentages in less good health for men and women separately for each of the 21 countries (data not shown here). Percentages in less good health were always higher for women than for men (for both WHSHSI and SRH). In nearly all countries differences between women and men were statistically significant (data not shown here). Differences by gender were largest in Portugal and Dominican Republic and smallest in the United Kingdom and Germany.

\section{WHSHSI and SRH by Age}

Fig. (1) shows the effect of age on WHSHSI and SRH for all respondents in 21 countries. With respect to WHSHSI it can be seen that the percentage in less good health increased monotonically with age up to and including age 80 years old.
The association of age with SRH was to some extent different; the percentage in less good health was smaller in the younger age groups and in the oldest age group (compared to WHSHSI). Differences between the two measures were minimal in the age groups 55-59, 60-64 and 65-69 years old. These differences in patterns between WHSHSI and SRH existed for both men and women (data not shown here).

\section{Comparison of WHSHSI and SRH with Life Expectancy at Age 20 by Gender}

We now compare the percentages in less good health (using both WHSHSI and SRH) with life expectancy at age 20 by gender (Fig. 2). In accordance with the finding mentioned earlier, the overall percentages in less good health were higher for women than for men (for both WHSHSI and SRH) (Panel A). For example, for all respondents the difference in less good health (using WHSHSI) was about 14 percentage points in favour of men (and the difference is statistically significant, $\mathrm{p}<0.001)$. In contrast, life expectancy at age 20 was higher for women than for men (calculated with the 21 countries as the unit of analysis) (Panel B); the difference in life expectancy by gender was about 4 years in favour of women (and this difference is statistically significant, $\mathrm{p}<0.05)$.

\section{Comparison of WHSHSI and SRH with Life Expectancy at Age 20 by Country Separately}

We also compared the rank orders of the two health status measures with life expectancy in each of the 21 countries. We are particularly interested to know to what extent the rank order of the two measures (WHSHSI and $\mathrm{SRH}$ ) corresponds with the rank order of the mortality measure. Fig. (3) shows the correspondence in the form of

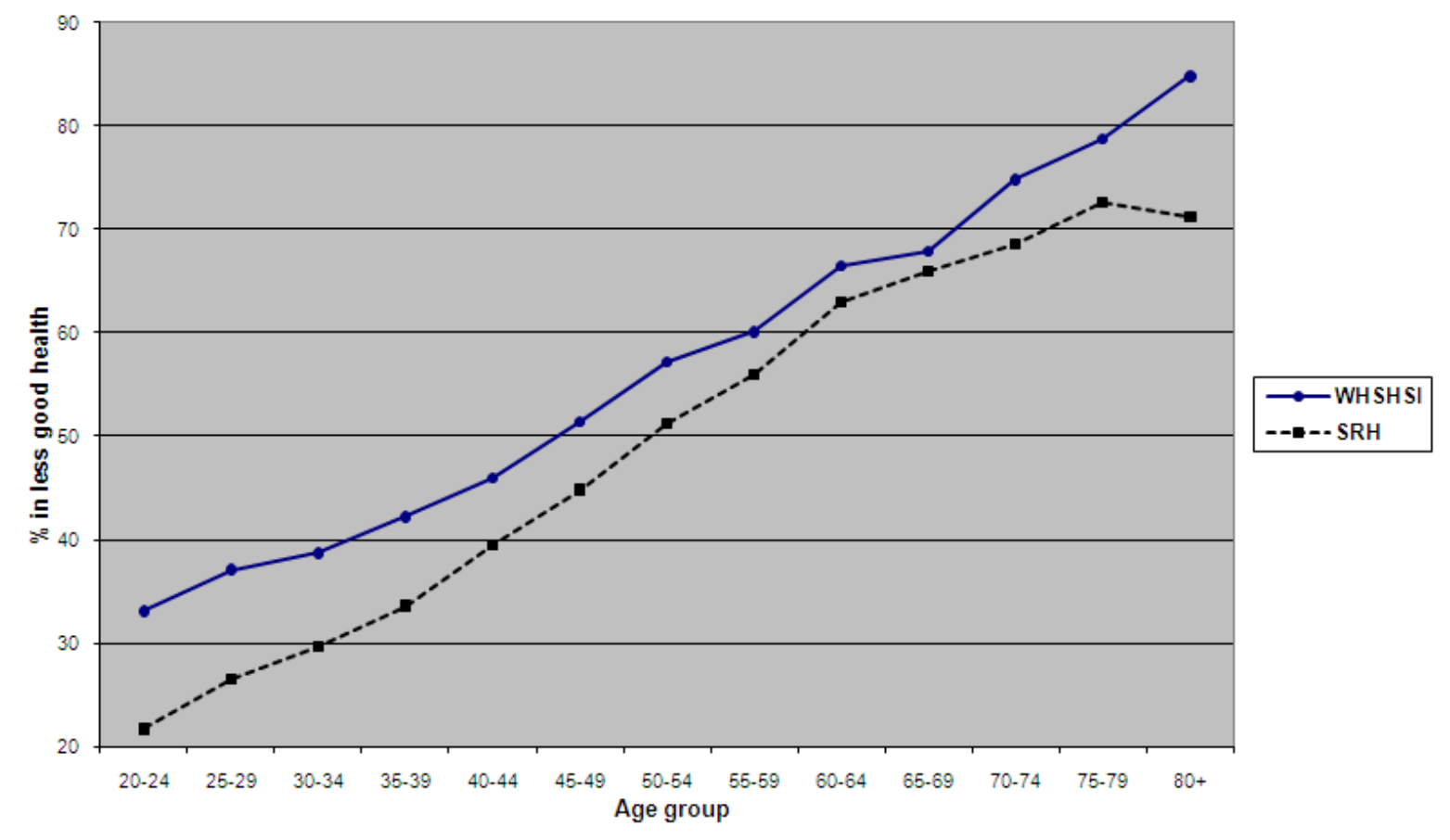

Fig. (1). Percentages in less good health according to two health measures, by age, average of 21 WHS countries. 


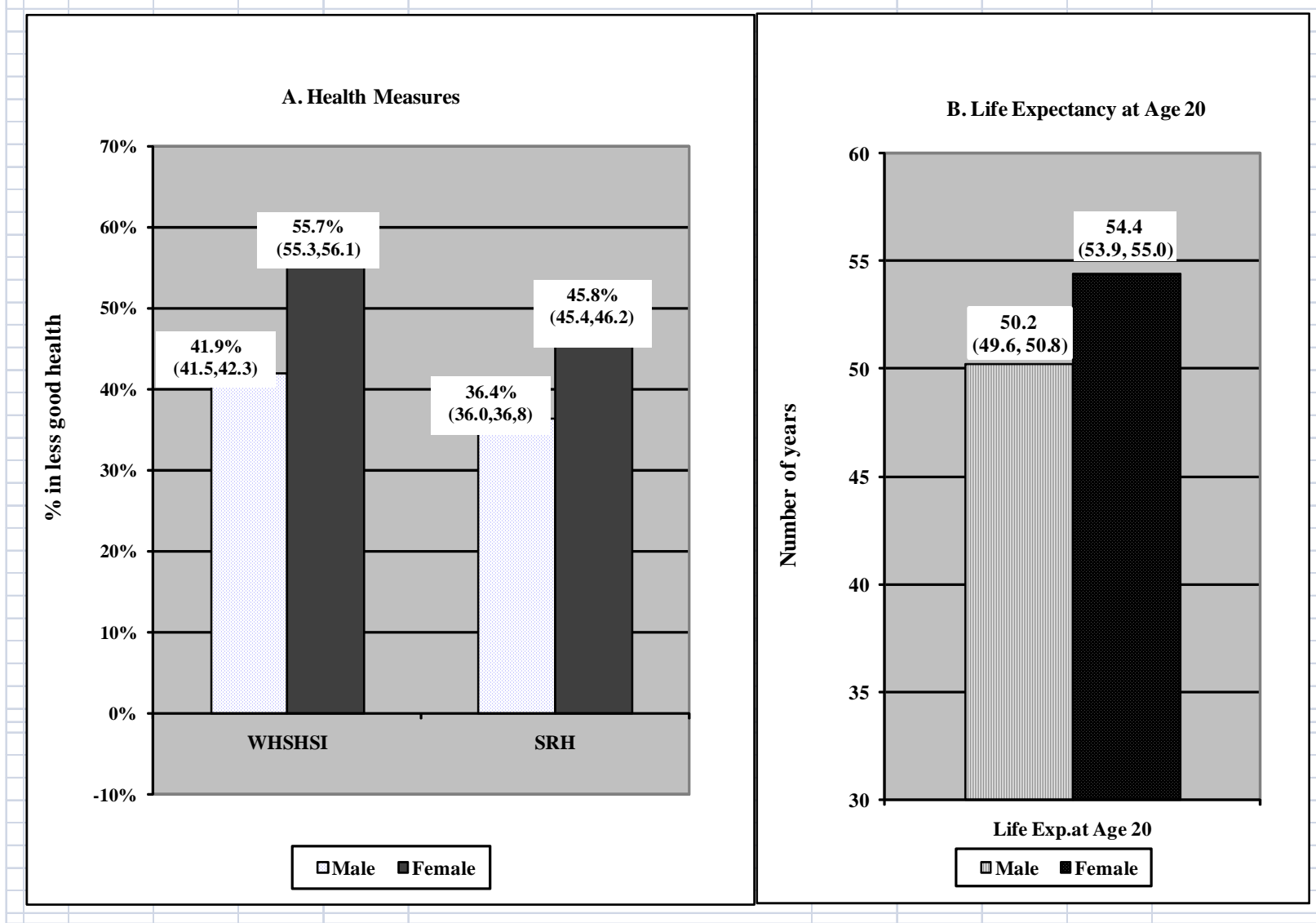

Fig. (2). Percentages in less good health by two health measures and life expectancy at age 20, average of 21 WHS countries.

deviations from zero. A value of zero denotes perfect agreement in the rank orders of percentages in less good health and life expectancy. A substantial deviation from zero means that there is considerable discrepancy in the rank orders between the two health measures and life expectancy.

Panel A of Fig. (3) shows that with respect to WHSHSI and life expectancy there is close correspondence for most of the 21 countries. The largest discrepancies between WHSHSI and life expectancy are found in four countries: Portugal, Brazil and Bangladesh (a higher percentage in less good health according to WHSHSI than according to life expectancy) and Ghana (a smaller percentage in less good health according to WHSHSI than according to life expectancy). Panel B of Fig. (3) shows to what extent there is similarity in the rank orders of SRH and life expectancy. The deviations from zero are on average much larger for SRH than for WHSHSI. There are in particular five countries that deviate most in the rank orders of SRH and life expectancy. Ghana, South Africa and Namibia report a much better health status according to SRH than one would expect on the basis of life expectancy values. On the other hand, Portugal and Vietnam report a much lower health status according to SRH than what would be expected on the basis of life expectancy levels.

We also noticed a pattern in the discrepancies in the ranking of SRH and life expectancy in two regions. All four African countries scored much higher on the SRH measure than on life expectancy. In other words, the respondents in these countries believed themselves to be in better health than levels of life expectancy suggest. The opposite pattern was noticed in the four Central and East European countries (especially Ukraine and Russia). Respondents in these countries were of the opinion that their health status was worse than we would expect based on life expectancy values.

\section{Correlation of WHSHSI and SRH with Life Expectancy for all 21 Countries Together}

The strength of the associations of the two health status measures with life expectancy at age 20 using correlation coefficients is shown in Table 2 . The correlation of the rank order of WHSHSI with life expectancy (with countries as the unit of analysis) is high and statistically significant (for example, 0.828 for both sexes). In other words, there is close correspondence in the rank orders of WHSHSI and life expectancy. Table 2 also shows that there is a fairly low correspondence between the rank orders of SRH and life expectancy (for example, correlation coefficients of 0.396 for both sexes and not significant).

Table 2 also shows the correlation of the rank orders of the WHSHSI and SRH variables. In general, the correlations are moderately high ( 0.542 for men and women together and statistically significant). This is an indication that to some extent there is overlap between what is measured by WHSHSI and SRH. Actually, the correspondence is close in most of the 21 countries, but there are three countries with low agreement between WHSHSI and SRH. These countries 
A. Rank-order differences between WHS Health Status Index (WHSHSI) and life-expectancy at age 20

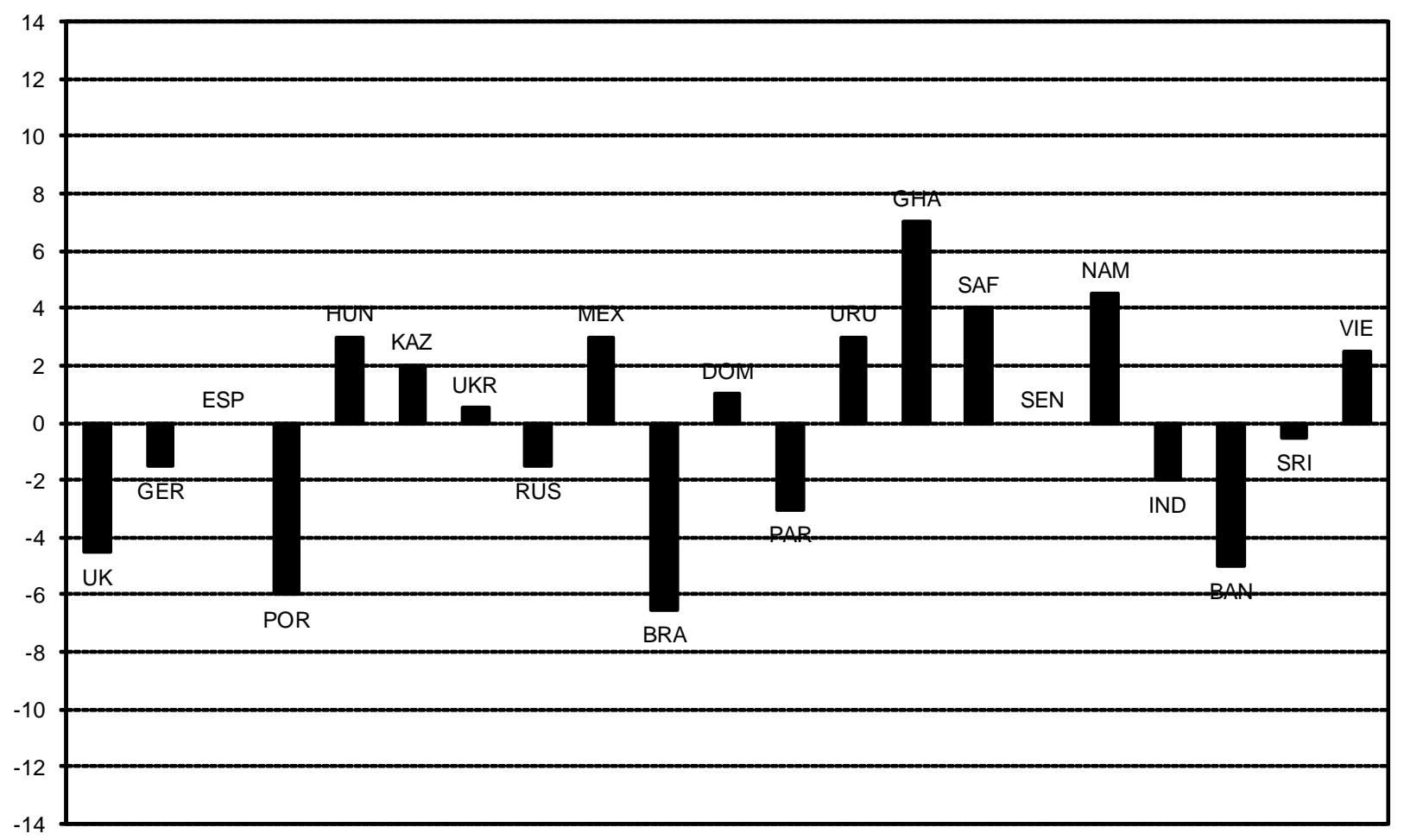

B. Rank-order differences between Self-Rated Health (SRH) and life-expectancy at age 20

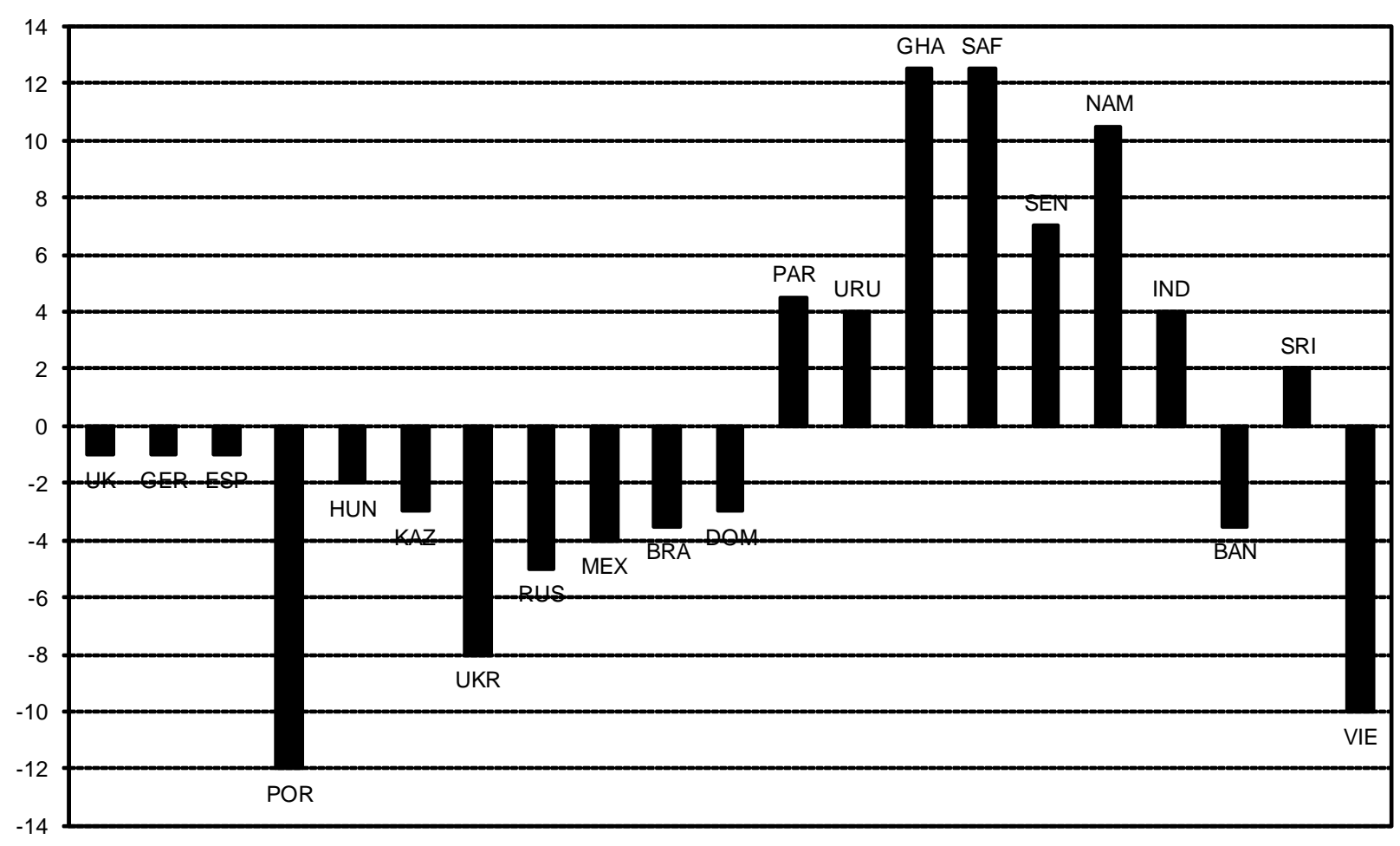

Fig. (3). Rank order differences between two health measures and life expectancy at age 20, 21 WHS countries.

are Vietnam, Ukraine and South Africa. Vietnam and Ukraine score low on WHSHSI (relative small percentages say they are in less good health), but very high on SRH (relative high percentages of respondents say they are in less good health). In South Africa we see the opposite pattern: this country scores high on WHSHSI (a high percentage of 
respondents say they are in less good health) and low on SRH (a small percentage of respondents say they in less good health).

Table 2. Spearman Rank Correlation Coefficients of Two Health Measures with Life Expectancy at Age 20, 21 WHS Countries

\begin{tabular}{|c|c|c|c|}
\hline & Male & Female & Total \\
\hline \hline WHSHSI with Life Expectancy & $0.813^{* *}$ & $0.800^{* *}$ & $0.828^{* *}$ \\
\hline SRI with Life Expectancy & 0.291 & 0.381 & 0.396 \\
\hline WHSHSI with SRI & $0.439 *$ & $0.521^{*}$ & $0.542^{*}$ \\
\hline Note: $* \mathrm{p}<0.05,{ }^{*} \mathrm{p}<0.001$ & \multicolumn{3}{|l}{}
\end{tabular}

\section{Correlation of WHSHSI and SRH with Chronic Conditions}

Table 3 shows the correlation coefficients of six chronic conditions ranging from arthritis to tuberculosis with the two health status measures (for all respondents and for men and women together). Correlations of all six conditions with the two measures are positive and statistically significant. Especially relevant for our study is the finding that correlations of the six chronic conditions with WHSHSI are higher than correlations of these conditions with SRH. With respect to diabetes, it can be seen that the difference between correlations is small although in the expected direction. As already mentioned earlier, the data on diabetes refer to 18 of the 21 countries. Table 3 also shows that the strength of the correlations coefficients of conditions with WHSHSI and SRH is moderate or small. For example, in the case of the relationships between conditions and WHSHSI the coefficients range from 0.370 to 0.150 .

Table 3. Pearson Correlation Coefficients of Symptoms of Selected Conditions with Two Health Measures, 21 WHS Countries

\begin{tabular}{|c|c|c|}
\hline $\begin{array}{c}\text { Symptoms of conditions } \\
\text { in past 12 months }\end{array}$ & $\begin{array}{c}\text { Conditions with WHS } \\
\text { Health Status Index }\end{array}$ & $\begin{array}{c}\text { Conditions with } \\
\text { Self Rated Health }\end{array}$ \\
\hline \hline Arthritis & $0.370^{* *}$ & $0.303^{* *}$ \\
\hline Angina Pectoris & $0.363^{* *}$ & $0.284^{* *}$ \\
\hline Asthma & $0.223^{* *}$ & $0.172^{* *}$ \\
\hline Depression & $0.342^{* *}$ & $0.225^{* *}$ \\
\hline Diabetes & $0.150^{* *}$ & $0.134^{* *}$ \\
\hline Tuberculosis & $0.170^{* *}$ & $0.130^{* *}$ \\
\hline Note: $* *$ p $<0.001$ &
\end{tabular}

\section{DISCUSSION}

\section{Percentages in Less Good Health (WHSHSI and SRH) by Age and Gender}

For all respondents together differences were observed in the shape of two curves depicting the associations of age with the two health measures. The most important difference occurred in the age group 80 years and older; the percentage in less good health according to WHSHSI continued to increase in this age group (compared to the age group 75-79 years old) while the percentage in less good health according to SRH decreased in this age group (compared to the age group 75-79 years old). It probably means that for respondents who were 80 years and older psychological and cultural factors played a more prominent role when replying to the SRH question than to the WHSHSI items. It could be that when they replied to the SRH question there was more of an opportunity to compare themselves with other persons in this age group and then concluded that they were better off than their peers. There was less of a chance to do this when replying to the 17 items that make up the WHSHSI index.

In all 21 countries women considered themselves on average to be in worse health than men (a larger percent of women said they were in less good health than men). This has already been found in several other studies conducted in high income countries (HICs) [7,12,23,24] and we showed that this also the case in a number of middle and low-income countries (MLICs).

\section{The Two Health Status Measures and Life Expectancy}

The strong association we found between WHSHSI and life expectancy at age 20 provides support for our thesis that this health measure is very useful in describing self-perceived morbidity in a variety of countries. We found, in contrast, a fairly weak association of SRH with life expectancy which implies that it is a less valid self-perceived morbidity measure than WHSHSI. In other words, at the country level there is a strong link between WHSHSI and mortality levels and a fairly weak link between SRH and mortality levels. This conclusion is based on the finding that, with respect to WHSHSI, in most of the 21 countries deviations in the rank orders of WHSHSI and life expectancy do not exist or are small. We also observed, however, that these deviations are more substantial in four countries. Deviations in the rank orders of WHSHSI and life expectancy are probably due to or influenced by specific cultural factors operating in these countries. It is likely that cultural factors play a more prominent role when using SRH. Not only were there more and larger deviations from zero in the majority of countries (in comparison with WHSHSI); in addition, in five countries correspondence with levels of life expectancy was very low.

These findings have implications, for instance, for longitudinal studies (with data available at the individual level) to be carried out on the predictive value of health status measures for subsequent mortality. We hypothesize that the predictive value of WHSHSI and other multi-item health status measures for subsequent mortality will be better than that of the single-item self-rated health measure.

\section{Role of Cultural and Psychological Factors}

An intriguing result was the discrepancy in the (ageadjusted) percentages in less good health between SRH and life expectancy in two regions. The contrast is particularly large between, on one hand, the African countries and, on the other hand, the Central and East European countries. Respondents in all four African countries consider themselves to be in better health according to the SRH than according to the level of life expectancy. In other words, they consider 
themselves to be in better health than the mortality figures imply. Exactly the opposite pattern is observed in four Central and East European countries; respondents in these countries believe that their state of health is worse (according to $\mathrm{SRH}$ ) than the level of life expectancy suggests.

Cultural reasons probably explain these differences in perception of health between the African and the Central and East European countries. There is evidence from a qualitative study conducted in Russia that living in that country is considered to be very stressful [23] and it could be that this stress has an influence on how people define and evaluate their health. Another study compared the health status in Russia with a few other countries. The unfavourable health status in Russia in comparison to other countries was attributed to the "dysfunctioning of social structures, socioeconomic deprivation and lack of perceived control" [25]. The same may apply to the other countries in that region. In African countries there may be a pattern of denial of the seriousness of health problems or the respondents in these countries may have a more optimistic attitude towards health than the mortality figures warrant. An indication that this could be the case is the pattern of denial on the existence of HIV/AIDS and related diseases that has been observed in South Africa [26]. This could also be the case in Namibia and, to a lesser extent, the other two African countries.

\section{The Two Health Measures and Self-Reported Chronic Conditions}

Higher correlations of five self-perceived chronic diseases with WHSHSI were observed than with SRH while there was only a small difference in the correlations with diabetes (but in the expected direction). It is another indication that WHSHSI, when compared with this second criterion, is more appropriate to use as a health measure than is SRH. A few studies have shown that SRH is related to chronic (and acute) conditions [9,27] and we believe, on the basis of our results, that such correlations would be stronger if WHSHSI had been used. It was noticed that correlations of the six conditions with WHSHSI and SRH were moderate or small. The reason for this is probably that each of these conditions, measured with only one question each, has a moderate or small impact on WHSHSI and SRH which are multi-dimensional concepts measuring perceived morbidity in general.

\section{Comparability of the Two Health Measures Across Countries}

We used similar cut-off points to define which of the respondents were in good or in less good health. For WHSHSI scores 9-10 indicated which persons were in less good health and for SRH corresponding scores were 3-5. The use of the same scoring system and cut-off points in all 21 countries has an important advantage. This means that it is possible to rank all respondents according to being in less good health using similar operational definitions. This increases comparability of measurement of health status across countries considerably. It is, however, not a perfect solution, because respondents in diverse socioeconomic, demographic and cultural settings may define and use response categories in different ways [17]. In order to increase comparability of health status measures across countries, WHS introduced anchoring vignettes, but until now they have only been applied on an experimental basis [28].

\section{Limitations}

A problem inherent in the type of research described here is that one cannot rule out that there are differences in quality of the data obtained in the 21 surveys. We described earlier the efforts that were made to obtain information of high quality. Nevertheless, it is possible that differences between countries were influenced by various sampling and nonsampling errors. Another frequently occurring problem in MLICs is the lack of accuracy on the reporting of the age of the respondents especially of older persons. Both problems have a similar effect on WHSHSI and SRH, but it limits cross-country comparability. Another limitation is that the WHS surveys were limited to the non-institutional populations. This is especially a problem in HICs with a high percentage of older people living in old-age and nursing homes and with other forms of in-patient care.

\section{CONCLUSION}

Data were used on two self-perceived morbidity measures, namely WHSHSI and SRH, in 21 countries included in the WHS project. WHSHSI was constructed using 17 items on functional limitations while SRH was derived from a single question frequently used in health surveys. We compared both measures with two criterion variables namely life expectancy and chronic conditions and found that WHSHSI was more strongly related to these two criterion variables than was SRH. It is, therefore, recommended to rely in health surveys as much as possible on WHSHSI and other multi-item health measures as indicators of self-perceived morbidity. There is thus scope for further development of valid and reliable multi-item self-perceived morbidity measures and in this respect we are more positive than Sen [15]. However, we agree with him that a self-perceived morbidity measure such as SRH has considerable limitations. It should only be used when other alternatives are not available, but researchers and policy makers should be aware of its restrictions.

We noticed earlier in this paper that data on the advantages and disadvantages of SRH are available for more surveys that are part of the WHS project than the 21 we used. It is, therefore, recommended to do more research on the validity of SRH and other health status measures using data from more WHS countries. Various health status data are also available from other types of survey programmes carried out in both HICs and MLICs. Datasets from these other survey programmes than WHS on SRH and other measures should also be analyzed.

It is recommended to apply new techniques to adjust for biases of the health status measures used. One is the already mentioned anchoring vignette approach [28] that could be useful for both multi-item and single-item measures. Two other closely-related techniques could be useful when dealing with biases in SRH. They consist of adjusting the values of different states of health varying from Very good to Very bad by means of weights derived from information on prevalence of chronic (and acute) conditions. These techniques can only be applied in situations in which there is 
information from the same surveys on both SRH and on prevalence of a number chronic (and acute) conditions $[8$, 29].

\section{CONFLICT OF INTEREST}

The authors declare that they do not have competing interests.

\section{ACKNOWLEDGEMENTS}

The authors are grateful to the World Health Survey of the World Health Organization for permission to make use of the data of the 21 countries included in this study.

\section{REFERENCES}

[1] Idler E, Benyamani Y. Self-rated health and mortality: a review of twenty-seven community studies. J Health Soc Behav 1997; 38: 21 37.

[2] McGee D, Liao Y, Cao G, Cooper S. Self-reported health status and mortality in a multiethnic US cohort. Am J Epidemiol 1999; 149: 41-8.

[3] Miilunpalo S, Vuori I, Oja P, Pasanen M, Urponen H. Self-rated health status as a health measure: the predictive value of selfreported health status on the use of physician services and on mortality in the working-age population. J Clin Epidemiol 1997; 50: 517-28.

[4] Fries J. Aging, natural death, and the compression of morbidity. N Engl J Med 1980; 3: 130-5.

[5] Crimmins E, Saito Y. Trends in healthy life expectancies in the United States, 1970-1990: gender, racial and educational differences. Soc Sci Med 2001; 52: 1629-41.

[6] Grigoriev P, Grigorieva O. Self-perceived health in Belarus: Evidence from the income and expenditures of household surveys. Demographic Res 2011; 24: 551-78.

[7] Mackenbach J, Stirbu I, Roskam A, et al. Socioeconomic inequalities in health in 22 European countries. N Engl J Med 2008; 358: 2468-81.

[8] Delpierre C, Lauwers-Cances V, Datta G, Lang T, Berkman L. Using self-rated health for analyzing social inequalities in health: a risk for underestimating the gap between socioeconomic groups. J Epidemiol Commun Health 2009; 63: 426-32.

[9] Kondo N, Sembajwe G, Kawachi I, van Dam R, Subramanian S, Yamagata Z. Income inequality, mortality, and self-rated health: meta-analysis of multilevel studies. Br Med J 2009; 339: 1178-81.

[10] Eriksson M, Dahlgren L, Janlert U, Weinehall L, Emmelin M. Social capital, gender and educational level - impact on self-rated health. Open Public Health J 2010; 3: 1-12.
[11] Subramaniam S, Huyts T, Avendano M. Self-reported health assessments in the 2002 world health survey: how do they correlate with education? Bull WHO 2010; 88: 131-8.

[12] Eriksson I, Unden A, Elofsson S. Self-rated health. Comparisons between three different measures. Results from a population study. Int J Epidemiol 2001; 30: 326-33.

[13] Jürges H, Avendano M, Mackenbach J. Are different measures of self-rated health comparable? An assessment in five European countries. Eur J Epidemiol 2008; 23: 773-81.

[14] Murray C, Chen L. Understanding morbidity change. Pop Dev Rev 1992; 18: 481-503.

[15] Sen A. Health: perception versus observation. Br Med J 2002; 324: 860-1.

[16] Subramanian S, Subramanyam M, Selvaraj S, Kawachi I. Are selfreports of health and morbidities in developing countries misleading? Evidence from India. Soc Sci Med 2009; 68: 260-5.

[17] Ustin T, Chatterjee S, Mechbal A, Murray C. The world health survey. In: Murray CJ, Evans DB, Eds. Health systems performance assessment: debates, methods and empiricism. Geneva: World Health Organization 2003; pp. 115-26.

[18] International Institute for Population Sciences and World Health Organization. Health System Performance Assessment, India, World Health Survey 2003. Mumbai International Institute for Population Sciences and Geneva: WHO 2006.

[19] Ustin T, Chatterjee S, Villnanueva M, et al. WHO multi-country survey study on health and responsiveness 2000-2001. In: Murray CJ, Evans DB, Eds. Health systems performance assessment: debates, methods and empiricism. Geneva: World Health Organization 2003; pp. 797-808.

[20] United Nations. World Population Prospects: The 2006 Revision. New York: United Nations 2007.

[21] Coale A, Demeny P. Regional model life tables and stable populations. New York: Academic Press 1983.

[22] Moussavi S, Chatterji S, Verdes E, Tandon A, Patel V, Ustun B. Depression, chronic diseases, and decrements in health: results from the world health surveys. Lancet 2007; 370: 851-8.

[23] Bobak M. Pikhart H. Hertzman C, Rose R, Marmot M. Socioeconomic factors, perceived control and self-reported health in Russia. A cross-sectional survey. Soc Sci Med 1998; 47: 269-79.

[24] Kunst A. Geurts J. van den Berg J. International variations in socioeconomic inequalities in self reported health. J Epidemiol Commun Health 1995; 49: 117-23.

[25] Pietila I, Rytkonen M. Coping with stress and by stress: Russian men and women talking about transition, stress and health. Soc Sci Med 2008; 66: 327-38.

[26] Barnett T, Whiteside A. AIDS in the twenty-first century: disease and globalization. Basingstoke: Palgrave MacMillan 2002.

[27] Rahman O, Barsky A. Self-reported health among older Bangladeshis: how good a health indicator is it? Gerontologist 2003; 43: 856-63.

[28] Salomon J, Tandon A, Murray C. Comparability of self-rated health: cross-sectional multi-country survey using anchoring vignettes. Br Med J 2004; 328: 258-64.

[29] Jürges H. True health $v s$. response styles: exploring cross-country differences in self-reported health. Health Econ 2007; 16: 163-78. 